BULLETIN OF THE

AMERICAN MATHEMATICAL SOCIETY

Volume 77, Number 4, July 1971

\title{
CHARACTERISTIC CLASSES FOR PL MICRO BUNDLES
}

\section{BY AKIHIRO TSUCHIYA ${ }^{1}$}

Communicated by M. H. Protter, December 9, 1970

0 . In this note we shall outline the results about the cohomology of BSPL, where BSPL is the classifying space of the stable oriented PL micro bundles. In this paper $p$ is always an odd prime number. The detailed version of these results will appear in [18].

THEOREM I. As a Hopf algebra over $Z_{p}$,

(i) $H_{*}\left(\mathrm{BSPL}: Z_{p}\right) \cong Z_{p}\left[b_{1}, b_{2}, \cdots\right] \otimes Z_{p}\left[\sigma\left(x_{I}\right)\right] \otimes \Lambda\left(\sigma\left(x_{J}\right)\right)$;

(ii) $\Delta\left(b_{j}\right)=\sum_{i=0}^{j} b_{i} \otimes b_{j-i}, b_{0}=1, \operatorname{deg} b_{j}=4 j$;

(iii) $\sigma\left(x_{I}\right), \sigma\left(x_{J}\right)$ are primitive elements.

TheOREM II. As a Hopf algebra over $Z[1 / 2]$,

(i) $H^{*}(\mathrm{BSPL}: Z[1 / 2]) /$ Torsions $=Z[1 / 2]\left[R_{1}, R_{2}, \cdots\right]$;

(ii) $\Delta R_{j}=\sum_{i=0}^{j} R_{i} \otimes R_{j}, R_{0}=1, \operatorname{deg} R_{j}=4 j$;

(iii) In $H^{*}(\mathrm{BSPL}: Q)=Q\left[p_{1}, p_{2}, \cdots\right], R_{j}$ are expressed as follows:

$$
R_{j}=2^{a_{j}}\left(2^{2 j-1}-1\right) \operatorname{Num}\left(B_{j} / 4 j\right) p_{j}+\operatorname{dec} \text { for some } a_{j} \in Z \text {. }
$$

Let MSPL denote the spectrum defined by the Thom complex of the universal PL micro bundles. Let $A=A_{p}$ denote the mod $p$ Steenrod algebra. $\phi: A \rightarrow H^{*}\left(\mathrm{MSPL}: Z_{p}\right)$ is defined by $\phi(a)=a(u)$, where $u \in H^{0}(\mathrm{MSPL})$ is the Thom class. The following is the conjecture of Peterson [12].

THEOREM III. The kernel of $\phi$ is $A\left(Q_{0}, Q_{1}\right)$, the left ideal generated by Milnor's elements $Q_{0}$ and $Q_{1}$.

1. The method to prove Theorem I is to compute the Serre spectral sequences associated to the fiberings, $\mathrm{SPL} \rightarrow \mathrm{SF} \rightarrow \mathrm{F} / \mathrm{PL} \rightarrow \mathrm{BSPL}$ $\rightarrow \mathrm{BSF}$. The structures of $H_{*}\left(\mathrm{SF}: Z_{p}\right)$ and $H_{*}\left(\mathrm{BSF}: Z_{p}\right)$ were determined in [9], [16] and [17]. The homotopy type of $F / P L$ is the deep result of Sullivan [15]. The first step is to study the $H$ space structure of $\mathrm{F} / \mathrm{PL}$ and the inclusion map $k: \mathrm{SF} \rightarrow \mathrm{F} / \mathrm{PL}$. The main tool in this step is the result of Sullivan [15], and its extension that tells the existence of the $\mathrm{KO}_{P}^{*}$ theory fundamental Thom classes for oriented $\mathrm{PL}$ micro bundles, where $\mathrm{KO}_{P}^{*}$ is 4 graded cohomology theory ob-

AMS 1970 subject classifications. Primary 55F40, 55F60.

Key words and phrases. Characteristic class, PL micro bundle, Steenrod algebra, fundamental Thom class.

1 The author was partially supported by the Sakkorkai Foundation. 
tained from localizing the ordinary $\mathrm{KO}^{*}$ theory at all odd primes $P$, cf. Sullivan [15].

Proposition 1-1. For any oriented PL disk bundle $\pi: E \rightarrow X$ over a finite complex $X$ of fiber dimension $m$, there exists the Thom class $u(\pi)$ $\in \mathrm{KO}^{m}(E, \partial E)_{P}$ with the following properties:

(i) Functorial, i.e. for $f: Y \rightarrow X, u\left(f^{\prime} \pi\right)=f^{\prime} u(\pi)$.

(ii) $\varphi_{H}^{-1} p h u(\pi)=L(\pi)^{-1} \in H^{*}(X, Q)$, where $\varphi_{H}$ is the Thom isomorphism, and $L(\pi)$ is the $L$ polynomial of Hirzebruch for $\pi: E \rightarrow X$.

(iii) $u(\pi \oplus 1)=\sigma u(\pi)$ for $\sigma: \mathrm{KO}^{m}(E, \partial E)_{P} \rightarrow \mathrm{KO}^{m+1}\left((E, \partial E) \wedge S^{1}\right)_{P}$, the suspension.

(iv) Multiplicative mod torsions, i.e. $u\left(\pi_{1} \oplus \pi_{2}\right)=u\left(\pi_{1}\right) \cdot u\left(\pi_{2}\right) \bmod$ torsion elements.

Let $\mathrm{BO}$ be the classifying space of the real vector bundles. This is a $H$ space defined by Whitney sum of bundles. Let $\mathrm{BO}\langle 8 N\rangle$ be the space obtained from $\mathrm{BO}$ by killing the homotopy groups $\pi_{i}(\mathrm{BO})$, $i<8 N$. Then by Bott periodicity $\Omega^{8 N} \mathrm{BO}\langle 8 N\rangle=\mathrm{BO} \times Z$, and $\mathrm{BO}$ and $\mathrm{BO} \times 0$ coincide as $H$ spaces. On the other hand there are products, $\mu_{M, N}: \mathrm{BO}\langle 8 M\rangle \times \mathrm{BO}\langle 8 N\rangle \rightarrow \mathrm{BO}\langle 8(M+N)\rangle$, obtained by tensor products of bundles. And we obtain the product $\mu: \Omega^{2 M} \mathrm{BO}\langle 8 M\rangle \times$ $\Omega^{8 N}\langle 8 N\rangle=(\mathrm{BO} \times Z) \times(\mathrm{BO} \times Z) \rightarrow \Omega^{8(M+N)} \mathrm{BO}\langle 8(M+N)\rangle=\mathrm{BO} \times Z$. Restricting $\mu$ to the 1 -component, we obtain a $H$ space $\mu_{\otimes}:(\mathrm{BO} \times 1)$ $\times(\mathrm{BO} \times 1) \rightarrow \mathrm{BO} \times 1$, and we denote this $H$ space by $\left(\mathrm{BO}_{\otimes}, \mu_{\otimes}\right)$. Then there is the natural homotopy equivalence $i: \mathrm{BO}=\mathrm{BO} \times 0 \rightarrow \mathrm{BO} \times 1$ $=\mathrm{BO}_{\otimes}$. Let $\mathrm{BO}_{P}$ and $\mathrm{BO}_{\otimes P}$ be the spaces obtained by localizing $\mathrm{BO}$ and $\mathrm{BO}_{\otimes}$ at all odd primes $P$. And $C_{P}$ denotes the class of abelian groups consisting of 2 -torsion groups. Then Sullivan [15] defined the $C_{P}$ homotopy equivalence

$$
\sigma: F / \mathrm{PL} \rightarrow \mathrm{BO}_{P}
$$

which is characterized by the formula,

$$
\sigma^{* *}\left(p h_{1}+p h_{2}+\cdots\right)=\frac{1}{8}\left(L_{1}+L_{2}+\cdots\right) \in H^{* *}(\mathrm{~F} / \mathrm{PL}, Q) .
$$

We define the map $\bar{\sigma}: \mathrm{F} / \mathrm{PL} \rightarrow \mathrm{BO}_{\otimes P}$ by

$$
\bar{\sigma}: \mathrm{F} / \mathrm{PL} \stackrel{\sigma}{\rightarrow} \mathrm{BO}_{P} \stackrel{\times 8}{\rightarrow} \mathrm{BO}_{P} \stackrel{i_{P}}{\rightarrow} \mathrm{BO} \otimes_{P} .
$$

Proposition 1-2. The $C_{P}$ homotopy equivalence $\bar{\sigma}$ is a $H$ space map.

Let $f_{N}: S^{8 N} \rightarrow \mathrm{BO}\langle 8 N\rangle$ be the representative of the canonical generator $\pi_{8 N}(\mathrm{BO}\langle 8 N\rangle) \cong Z$. Then we obtain the map $g: \Omega^{8 N} S^{8 N} \rightarrow \mathrm{BO}$ $\times Z$, and passing to the limit, $g: Q S^{0} \rightarrow \mathrm{BO} \times Z$, where $Q S^{0}=\lim \Omega^{8 N} S^{8 N}$. The 1-component $Q_{1} S^{0}$ of $Q S^{0}$ becomes a $H$ space by reduced join 
product and this $H$ space is equivalent to $\mathrm{SF}$. So we obtain a $H$ map $g_{1}: \mathrm{SF} \rightarrow \mathrm{BO}_{\otimes}$.

Proposition 1-3. The maps $g_{1}: \mathrm{SF} \rightarrow \mathrm{BO}_{\otimes} \rightarrow \mathrm{BO}_{\otimes P}$, and $\bar{\sigma} \circ k: \mathrm{SF}$ $\rightarrow \mathrm{F} / \mathrm{PL} \rightarrow \mathrm{BO}_{\otimes P}$ coincide up to homotopy.

Corollary 1-4. As a Hopf algebra over $Z_{p}, H_{*}\left(\mathrm{~F} / \mathrm{PL}: Z_{p}\right)$ $=Z_{p}\left[a_{1}, a_{2}, \cdots\right] . \Delta a_{j}=\sum_{i=0}^{j} a_{i} \otimes a_{j-i}, a_{0}=1 . \operatorname{deg} a_{j}=4 j$.

Then Theorem I is obtained by tediously long calculations using Proposition 1-3, and results of [17] about $H_{*}(\mathrm{SF})$ and $H_{*}(\mathrm{BSF})$.

2. The first step to prove Theorem II is to compute the Bockstein spectral sequence, with $E_{*}^{1}=H_{*}\left(\mathrm{BSPL}: Z_{p}\right)$ and $E_{*}^{\infty}$ $=\left(H_{*}(\mathrm{BSPL}: Z) /(\right.$ Torsions $\left.)\right) \otimes Z_{p}$. And then studying the map $\left(H_{*}(\mathrm{~F} / \mathrm{PL}: Z) /\right.$ Torsion $) \otimes Z_{p}$

$$
=H_{*}\left(\mathrm{~F} / \mathrm{PL}: Z_{p}\right) \rightarrow\left(H_{*}(\mathrm{BSPL}: Z) / \text { Torsions }\right) \otimes Z_{p},
$$

and

$\left(H_{*}(\mathrm{BSO}: Z) /\right.$ Torsions $) \otimes Z_{p} \rightarrow\left(H_{*}(\mathrm{BSPL}: Z) /\right.$ Torsions $) \otimes Z_{p}$, we obtain Theorem II.

3. The essential part to prove Theorem III is the following proposition.

Proposition 3-1. There exists a oriented PL disk bundle $\pi: E \rightarrow X$ for some $X$, with the following properties:

$Q_{j}(u) \neq 0$ for $j \geqq 2$, where $u \in H^{*}\left(E, \partial E: Z_{p}\right)$ is the Thom class and $Q_{j}$ are the Milnor elements.

The construction of $\pi: E \rightarrow X$ is the following. Let $K$ be a CW complex of the form,

$$
K=S^{p r-1} \cup_{p} e^{p r} \cup_{\alpha_{1}} e^{(p+1) r} \cup_{p} e^{(p+1) r+1}, \quad r=2(p-1),
$$

and let $f: K \rightarrow \mathrm{BSPL}$ be the map which represents $\beta_{1}$ in $j \circ f \circ i: S^{p r-1}$ $\rightarrow K \rightarrow \mathrm{BSPL} \rightarrow \mathrm{BSF}$. Then $f$ is represented by a PL disk bundle $\pi_{f}: E_{f} \rightarrow K$ of fiber dimension $N, N \gg 0$. Let $\pi_{p}$ be the cyclic group of order $p$, and $W\left(\pi_{p}\right)=W$ be the free $\pi_{p}$ acyclic complex. Then $P\left(\pi_{f}\right): W$ $\times_{\pi_{p}}\left(E_{f}\right)^{p} \rightarrow W \times_{\pi_{p}}(K)^{p}$ is a oriented PL disk bundle of fiber $\operatorname{dim} p N$. This is the bundle we seek.

\section{REFERENCES}

1. J. F. Adams, On the groups $J(X)$. II, Topology 3 (1965), 137-171. MR 33 \#6626.

2. S. Araki and $\mathrm{H}$. Toda, Multiplicative structures in $\bmod q$ cohomology theories. I, Osaka J. Math. 2 (1965), 71-115. MR 32 \#449. 
3. M. Atiyah and F. Hirzebruch, Riemann-Roch theorems for differentiable manifolds, Bull. Amer. Math. Soc. 65 (1959), 276-281. MR 22 \#989.

4. G. E. Bredon, Equivariant cohomology theories, Lecture Notes in Math., no. 34, Springer-Verlag, Berlin and New York, 1967. MR 35 \#4914.

5. W. Browder, Homotopy commutative H-spaces, Ann. of Math. (2) 75 (1962), 283-311. MR $27 \# 765$.

6. G. Brumfiel, On integral PL characteristic classes, Topology 8 (1968), 39-46. MR 38 \#2801.

7. P. E. Conner and E. E. Floyd, Differentiable periodic maps, Ergebnisse der Mathematik und ihrer Grenzgebiete, Heft 33, Springer-Verlag, Berlin, 1964. MR 31 \#750.

8. E. Dyer and R. K. Lashof, Homology of iterated loop spaces, Amer. J. Math. 84 (1962), 35-88. MR 25 \#4523.

9. P. May, The homology of $F, F / O, B F$ (to appear).

10. J. W. Milnor, The Steenrod algebra and its dual, Ann. of Math. (2) 67 (1958), 150-171. MR $20 \# 6092$.

11. G. Nishida, Cohomology operations in iterated loop spaces, Proc. Japan Acad. 44 (1968), 104-109. MR 39 \#2156.

12. F. P. Peterson, Some results in PL cobordism, J. Math. Kyoto Univ. 9 (1969), 189-194. MR 40 \#4962.

13. F. P. Peterson and $\mathrm{H}$. Toda, $O n$ the structure of $H^{*}\left(\mathrm{BSF}: Z_{p}\right)$, J. Math. Kyoto Univ. 7 (1967), 113-121. MR 37 \#5878.

14. D. Sullivan, Triangulating homotopy equivalence, Thesis, Princeton University, Princeton, N. J., 1966.

15. - Geometric topology seminar note.

16. A. Tsuchiya, Characteristic classes for spherical fiber spaces, Proc. Japan Acad. 44 (1968), 617-622. MR 40 \#2115.

17. —- Characteristic classes for spherical fiber spaces, Nagoya Math. J. 43 (to appear).

18. - Characteristic classes for PL micro bundles, Nagoya Math. J. 43 (to appear).

19. - Homology operations on iterated loop spaces (to appear).

NAgoya University, NAGOYa, Japan 